\title{
Structural differences in the brain between wild and laboratory rats (Rattus norvegicus): Potential contribution to wariness
}

\author{
Ryoko KOIZUMI ${ }^{1)}$, Yasushi KIYOKAWA ${ }^{1) *}$, Kaori MIKAMI'), Akiko ISHII1), \\ Kazuyuki D. TANAKA ${ }^{2)}$, Tsutomu TANIKAWA ${ }^{2)}$ and Yukari TAKEUCHI ${ }^{1)}$ \\ 1)Laboratory of Veterinary Ethology, The University of Tokyo, 1-1-1 Yayoi, Bunkyo-ku, Tokyo 113-8657, Japan \\ ${ }^{2)}$ Technical Research Laboratory, Ikari Shodoku Corporation, 1-12-3 Akanehama, Narashino-shi, Chiba 275-0024, \\ Japan
}

J. Vet. Med. Sci.

80(7): 1054-1060, 2018

doi: 10.1292/jvms.18-0052

Received: 1 February 2018

Accepted: 1 May 2018

Published online in J-STAGE: 11 May 2018

\begin{abstract}
Wild animals typically exhibit defensive behaviors in response to a wider range and/or a weaker intensity of stimuli compared with domestic animals. However, little is known about the neural mechanisms underlying "wariness" in wild animals. Wild rats are one of the most accessible wild animals for experimental research. Laboratory rats are a domesticated form of wild rat, belonging to the same species, and are therefore considered suitable control animals for wild rats. Based on these factors, we analyzed structural differences in the brain between wild and laboratory rats to elucidate the neural mechanisms underlying wariness. We examined wild rats trapped in Tokyo, and weight-matched laboratory rats. We then prepared brain sections and compared the basolateral complex of the amygdala (BLA), the bed nucleus of the stria terminalis (BNST), the main olfactory bulb and the accessory olfactory bulb. The results revealed that wild rats exhibited larger BLA, BNST and caudal part of the accessory olfactory bulb compared with laboratory rats. These results suggest that the BLA, BNST, and vomeronasal system potentially contribute to wariness in wild rats.
\end{abstract}

KEY WORDS: accessory olfactory bulb, basolateral complex of the amygdala, bed nucleus of the stria terminalis, main olfactory bulb, vomeronasal receptor

Defense is one of the fundamental characteristics of animal. Animals detect environmental stimuli via sensory systems, evaluate whether the stimuli are harmful, and exhibit specific defensive behaviors in response to stimuli judged as threatening. Comparisons between wild and domestic animals have suggested the presence of a similar repertoire of defensive behaviors, but marked differences in the expression of these behaviors. In general, wild animals exhibit defensive behaviors in response to a wider range and/or a weaker intensity of stimuli compared with domestic animals. For example, a previous study reported that plains zebras showed flight responses to an approaching human sooner than domesticated feral horses [3]. The term "wariness" is operationally used in the current study to refer to this characteristic. However, little is known about the neural mechanisms underlying wariness in wild animals.

Wild rats (Rattus norvegicus) are among the most accessible wild animals for experimental research because they have been major pests in human societies for thousands of years [21]. Wild rats appear to exhibit a high level of wariness, which has enabled them to survive against numerous attempts to eliminate them. This is exemplified by the heightened tendency of wild rats to avoid novel objects. In one study, when a small piece of wood was suddenly placed between the entrance hole of a shed and a familiar baiting station in the shed, wild rats were observed to stop at the entrance hole, frequently raising their noses high into the air, then withdraw [4]. Given that the descendants of wild rats born and reared in the laboratory show a similar intensity of avoidance [6, 9], genetic factors appear to be the predominant determinants of wariness, compared with environmental factors. Laboratory rats are a domesticated form of wild rat, and are considered suitable control animals for wild rats. They are major experimental animals, and have provided a substantial amount of knowledge about the relationship between the brain and behavior [36]. In addition, laboratory rats are the same species as wild rats, having the same binomial name and the ability to produce hybrids [1]. These factors propose that wild and laboratory rats can be effective experimental models for investigating wariness in wild animals.

Research on laboratory rats has identified several brain regions that participate in defensive behaviors, including the basolateral complex of the amygdala (BLA). For example, a lesion or inactivation of the BLA in laboratory rats has been found to dramatically reduce flight responses to an approaching predator-like robot [5], and to decrease freezing in response to a tone

*Correspondence to: Kiyokawa, Y.: akiyo@mail.ecc.u-tokyo.ac.jp

O2018 The Japanese Society of Veterinary Science

This is an open-access article distributed under the terms of the Creative Commons Attribution Non-Commercial No Derivatives (by-nc-nd) License. (CC-BY-NC-ND 4.0: https://creativecommons.org/licenses/by-nc-nd/4.o/) 
that was associated with a foot shock [16]. The bed nucleus of the stria terminalis (BNST) has also been linked with defensive behaviors. For example, previous studies have reported that a lesion or inactivation of the BNST blocked an enhancement of the acoustic startle reflex in response to bright light illumination [35], or freezing to a test box in which the laboratory rats received foot shocks [16]. Not limited to these examples, a large body of evidence supports the notion that the BLA [18] and BNST [10, 30] play pivotal roles in defensive behaviors. Based on these findings, it is possible that the BLA and/or BNST are activated in wild rats in response to a wider range and/or weaker intensity of stimuli. In other words, it is possible that the BLA and/or BNST are more sensitive to threat stimuli in the wild compared with laboratory rats.

Neuronal activity in these nuclei is likely to be linked with size. For example, one study reported that when exposure to stressors developed the BNST-mediated response (increased time spent in open arms in the elevated plus maze), but not the BLA-mediated response (fear-potentiated startle reflex), laboratory rats exhibited enlargement of the BNST, but not the BLA [25]. These findings suggest that the size of brain nuclei can be used to infer general neural activity. Based on this assumption, in the current study, we hypothesized that wild rats would exhibit larger BLA and/or BNST compared with laboratory rats.

Previous studies have also revealed that olfactory signals can induce defensive behaviors in laboratory rats. For example, predator odor has been widely used as a stimulus for inducing defensive behaviors [8,31,32]. In addition, an olfactory signal released from distressed conspecifics known as the alarm pheromone is reported to be sufficient to induce a wide variety of defensive behaviors [12, 15]. Furthermore, involvement of the BNST [2, 7] and BLA [33] in these defensive behaviors has been reported. Based on these findings, it is possible that wild rats exhibit superior olfactory ability compared with laboratory rats, enabling them to detect a wider range and/or weaker intensity of olfactory threats.

In the olfactory system, odorants are detected by receptors expressed at the sensory neurons. Therefore, the olfactory ability of an animal can be evaluated by the number of sensory neurons and/or the number of receptor genes. The olfactory system is known to contain two major systems: the main olfactory system and the vomeronasal system [19, 22]. In the main olfactory system, the main olfactory bulb (MOB) is enveloped by glomeruli that each receive projections from multiple sensory neurons, but which express a single olfactory receptor at the main olfactory epithelium. Therefore, the number of glomeruli in each olfactory bulb represents the number of olfactory receptor genes, which can be used as an index of the functioning of the main olfactory system. In the vomeronasal system, there are two types of vomeronasal receptors: V1R and V2R. In contrast to the main olfactory system, sensory neurons expressing a single V1R or V2R at the vomeronasal epithelium project to multiple glomeruli of the accessory olfactory bulb (AOB). However, the projections from neurons expressing V1R and V2R are restricted within the rostral and caudal parts of the AOB, respectively. In addition, the boundary of each glomerulus at the AOB is less obvious compared with glomeruli at the MOB. Therefore, it would be reasonable to infer the level of functioning of the vomeronasal system using V1R and V2R, by measuring the size of the rostral and caudal parts of the glomerulus layer (GL) of the AOB, respectively. Based on these anatomical findings, we hypothesized that wild rats would have a larger number of glomeruli in the MOB and/or a larger AOB compared with laboratory rats.

To test these hypotheses, we trapped wild rats at a market in Tokyo, and sampled brain tissue. In addition, we prepared weightmatched laboratory rats, and sampled brain tissue. We then compared the size of the BLA, BNST, MOB, glomeruli in the MOB and GL in the AOB.

\section{MATERIALS AND METHODS}

All experiments were approved by the Animal Care and Use Committee of the Faculty of Agriculture at The University of Tokyo, according to guidelines adapted from the Consensus Recommendations on Effective Institutional Animal Care and Use Committees by the Scientists Center for Animal Welfare.

\section{Animals}

Wild rats (seven males and three females) were captured at a market in Tokyo, Japan and transferred immediately to the laboratory at Ikari Shodoku Corporation. We purchased weight-matched Wistar rats (seven males: 6 weeks old, three females: 8 weeks old) from Charles River Laboratories Japan (Yokohama, Japan) for use as laboratory rats. Upon arrival, they were kept individually in a wire-mesh cage $(23 \times 14 \times 10 \mathrm{~cm})$ in a room with an ambient temperature of $20 \pm 5^{\circ} \mathrm{C}$ and a 12 -hr light/dark cycle (lights were switched on at 8:00). Food (CE-2, Clea Japan, Tokyo, Japan) and water were available ad libitum. Wild rats were confirmed as brown rats (Rattus norvegicus) based on their morphological characteristics such that the ears were not large enough to reach to the eyes when folded and the tail was shorter than the body length. In addition, our informal observations confirmed differences in wariness between the wild and laboratory rats. For example, when an experimenter approached the home cage, wild rats escaped the far side of the cage. In contrast, the laboratory rats did not respond, or even came closer to the experimenter.

\section{Analyses of general information}

Analyses of general information were conducted using the method reported in our previous study [17]. To minimize the effects of housing in the laboratory, brain tissue was sampled within 1 or 2 days after arrival. All subjects were deeply anesthetized with sodium pentobarbital and weighed. Subjects were then intracardially perfused with saline followed by $4 \%$ paraformaldehyde in 0.1 M phosphate buffer. Sampled brains were vertically cut at the caudal end of the cerebellum and weighed. Body and brain weights were analyzed using Student's $t$-tests. Additional analyses on bodies and brains are provided in the Supplemental Materials. 


\section{Immunohistochemistry}

After being kept in the same fixative overnight, sampled brains were placed in $30 \%$ sucrose/phosphate buffer for cryoprotection. We prepared six BLA-containing coronal sections $(50 \mu \mathrm{m})$ every five sections from the bregma $-3.48 \mathrm{~mm}$ to the anterior. We also prepared six consecutive BNST-containing coronal sections $(50 \mu \mathrm{m})$ from the bregma $-0.12 \mathrm{~mm}$ to the posterior. Sagittal sections of the olfactory bulb were prepared by collecting every four AOB-containing sections $(50 \mu \mathrm{m})$ from the lateral to medial, obtaining as many sections as possible.

Immunohistochemistry for neuronal nuclei $(\mathrm{NeuN})$ protein was conducted as previously described [17]. After processing with $0.3 \% \mathrm{H}_{2} \mathrm{O}_{2}$ in phosphate buffered saline, the sections were incubated with citric acid buffer (LSI Medience, Tokyo, Japan) for $2 \mathrm{hr}$, followed by incubation with a primary antibody to NeuN protein (MAB377; Merck Millipore, Billerica, MA, U.S.A.) overnight, then with biotinylated anti-mouse secondary antibody (BA-2000; Vector Laboratories, Burlingame, CA, U.S.A.) for $2 \mathrm{hr}$. Finally, sections were processed with the VECTASTAIN Elite ABC kit (Vector Laboratories) and developed using a diaminobenzidine solution with nickel intensification. The AOB-containing sections were further incubated with primary antibody for G protein Gia-2 (MAB3077; Merck Millipore) overnight, incubated with biotinylated anti-mouse secondary antibody (BA2000; Vector Laboratories) for $2 \mathrm{hr}$, processed with the VECTASTAIN Elite ABC kit (Vector Laboratories), and developed using a diaminobenzidine solution.

\section{Analyses of the brain nuclei}

Images of sections were captured using a microscope equipped with a digital camera (DP30BW, Olympus, Tokyo, Japan). An experimenter who was blinded to the subject group analyzed the areas of interest using ImageJ 1.45s software. To compare the size of the BLA and BNST, we measured the area of the whole section and the area of the nucleus bilaterally. We also counted the number of immunoreactive cells in the BLA and BNST bilaterally. In the AOB-containing sections, we measured an area of 30 to 72 glomeruli in the anterodorsal region of the MOB bilaterally in each subject. We also measured the length of the outer line of the external plexiform layer (EPL) of the MOB in four to 11 sections bilaterally in each subject. To compare the areas of the rostral and caudal part of the AOB, the GL of the AOB was divided into a Gi $\alpha-2$ expressing rostral region and the remaining caudal regions. The areas of the rostral part, caudal part, and whole GL of the AOB were measured in four to 11 sections bilaterally in each subject. All of these data were analyzed using Student's $t$-tests.

\section{RESULTS}

Data are expressed as the mean \pm standard error of the mean. $P$-values $<0.05$ were considered significant for all statistical analyses.

First, we confirmed that body weight was not significantly different between the wild and laboratory rats. Sampling brain tissue revealed that brain weight was not significantly different between wild and laboratory rats (Table 1). Additional analyses are provided in the Supplemental Materials.

To compare BLA size, we prepared BLA-containing coronal sections and used immunohistochemical staining for NeuN as a marker for neurons (Fig. 1A). We found that the BLA area of wild rats was significantly larger than that of laboratory rats $(P<0.01)$, while the area of the whole section was not significantly different (Fig. 1B and 1C). To assess whether the enlargement of the area was accompanied by an increase in the number of neurons, we counted the number of NeuN-immunoreactive cells in the BLA and calculated the neuronal density. We found that the density of neurons was higher in the wild rats than in the laboratory rats $(P<0.01$, Fig. 1D). Taken together, these results suggest that the BLA was larger in the wild rats than in the laboratory rats.

We prepared BNST-containing coronal sections to conduct the same analyses (Fig. 2A). We found that the BNST area was larger in the wild rats than in the laboratory rats $(P<0.05)$ while the area of the whole section was not significantly different (Fig. 2B and $2 \mathrm{C}$ ). In the BNST, the density of neurons was not significantly different between the wild and laboratory rats (Fig. 2D). Taken together, these results suggest that the BNST was larger in wild rats compared with laboratory rats.

Because it is infeasible to count the number of the glomeruli in the MOB, we rationally compared the number of glomeruli by measuring the sizes of the olfactory bulb and glomeruli. For example, if one group had a larger olfactory bulb with similarly sized glomeruli, the greater surface area would be expected to be covered with similar sized glomeruli. Thus, the number of glomeruli would logically be higher in this group. When we compared the olfactory bulb, the length was greater in wild rats $(P<0.01)$ whereas the maximum breadth was wider in laboratory rats $(P<0.05)$ (Table 2$)$, preventing us from confirming which group had larger olfactory bulbs. To compare the size of the olfactory bulb in more detail, we prepared AOB-containing sagittal sections. We then immunohistochemically stained the sections for NeuN and G protein Gia-2 and compared the length of the outer line of the EPL of the MOB. Because the glomeruli were arranged on the surface of the EPL, the length is thought to provide an index of the surface area of the olfactory bulb. We found that the length was no different between the wild and laboratory rats when we measured four to 11 sections in each subject (Table 2). In addition, when we measured the area of 30 to 72 glomeruli in each subject, we found no significant difference between wild and laboratory rats (Table 2). Taken together, these findings indicated that the number of glomeruli in the MOB was similar between wild and laboratory rats.

When we compared the AOB (Fig. 3A), we found that the area of whole GL was significantly larger in wild rats compared with laboratory rats $(P<0.01)$

Table 1. Body weight and brain weight

\begin{tabular}{lcc}
\hline & Wild & Laboratory \\
\hline Body weight $(\mathrm{g})$ & $173 \pm 11$ & $189 \pm 4$ \\
Brain weight $(\mathrm{g})$ & $1.72 \pm 0.04$ & $1.70 \pm 0.02$ \\
\hline
\end{tabular}


a

\section{BLA}

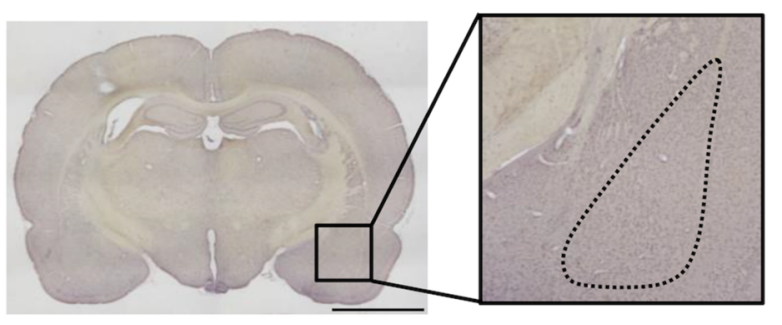

Wild

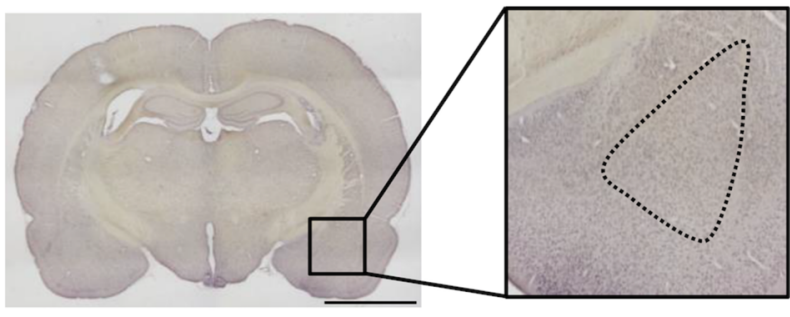

Laboratory

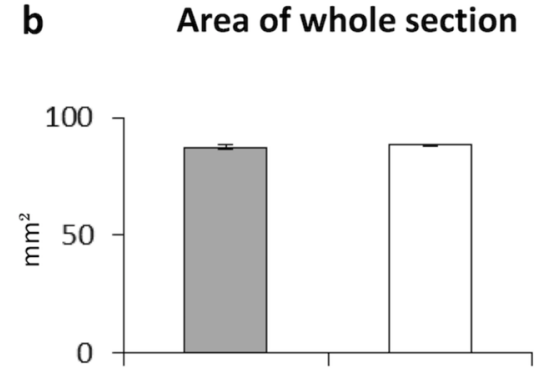

Wild

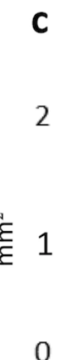

2
1
0
Area of nucleus

$*$

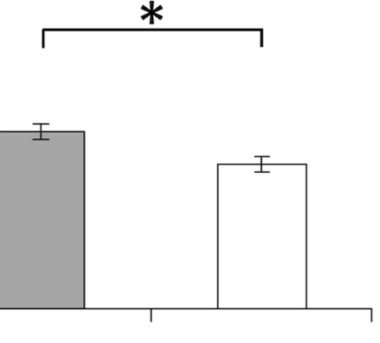

Wild

Laboratory

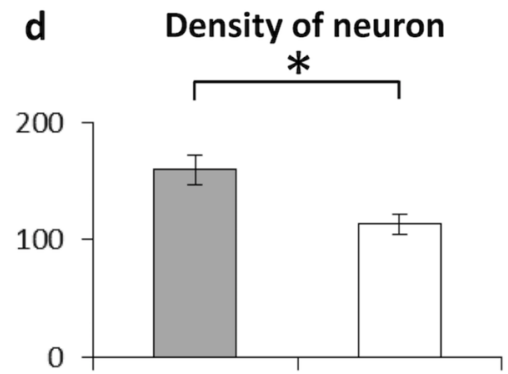

Wild

Laboratory

Fig. 1. Anatomical analyses of the basolateral complex of the amygdala (BLA). (A) A representative BLA-containing section photograph of wild and laboratory rats. The horizontal bar shows $4 \mathrm{~mm}$. The area of (B) whole section and (C) nucleus and (D) density of neurons in the wild (Wild) and laboratory rats (Laboratory). ${ }^{*} P<0.05$ with Student's $t$-test.

a

BNST

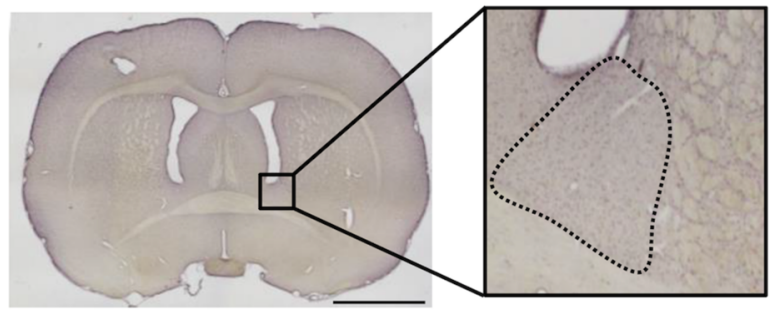

Wild

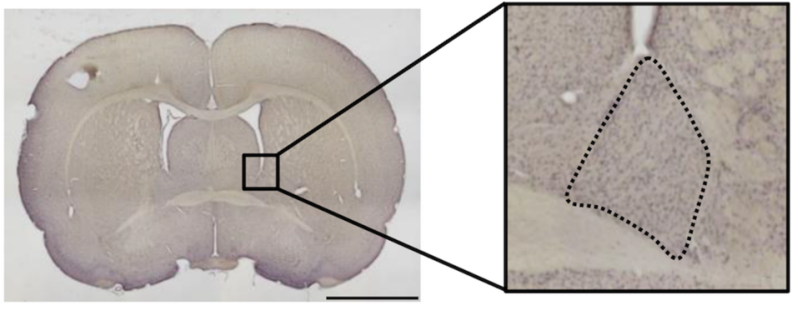

Laboratory b

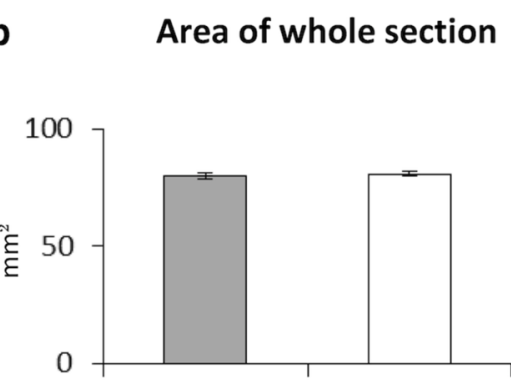

Wild
0

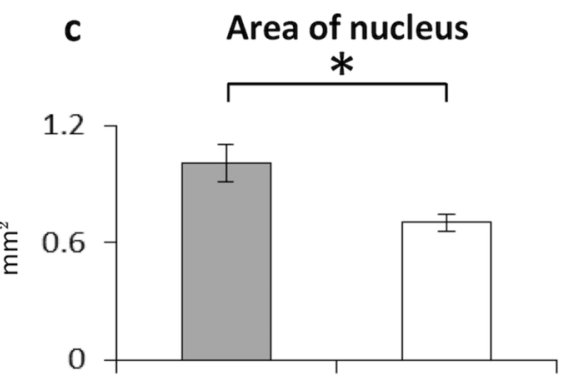

Area of nucleus

$*$

Wild

Laboratory

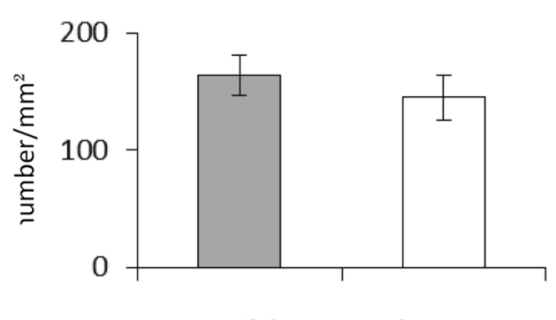

Fig. 2. Anatomical analyses of the bed nucleus of the stria terminalis (BNST). (A) A photograph of the representative BNST-containing section of wild and laboratory rats. The horizontal bar shows $4 \mathrm{~mm}$. The area of (B) whole section and (C) nucleus and (D) density of neurons in wild (Wild) and laboratory rats (Laboratory). $* P<0.05$ with Student's $t$-test. 
Table 2. Comparisons in the main olfactory bulb

\begin{tabular}{lcc}
\hline & Wild & Laboratory \\
\hline Length of the olfactory bulb $(\mathrm{mm})$ & $4.8 \pm 0.2^{\text {a) }}$ & $3.7 \pm 0.1$ \\
Greatest breadth of the olfactory bulb (mm) & $4.5 \pm 0.1^{\text {a) }}$ & $4.8 \pm 0.1$ \\
Area of the glomerulus $\left(\mu \mathrm{m}^{2)}\right.$ & $11,172 \pm 375$ & $10,922 \pm 333$ \\
Length of outer line of the EPL (mm) & $1.38 \pm 0.05$ & $1.34 \pm 0.03$ \\
\hline
\end{tabular}

a) $P<0.05$ with Student's $t$-test.

a

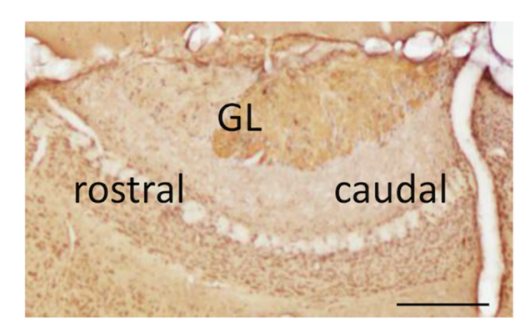

Wild

\section{$\mathrm{AOB}$}

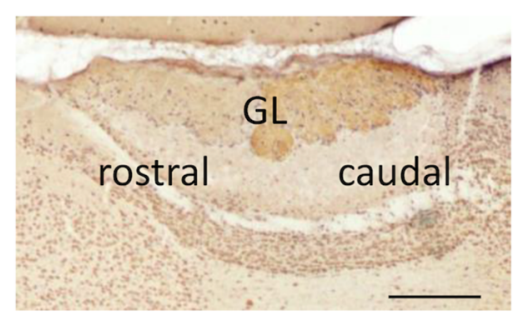

Laboratory b

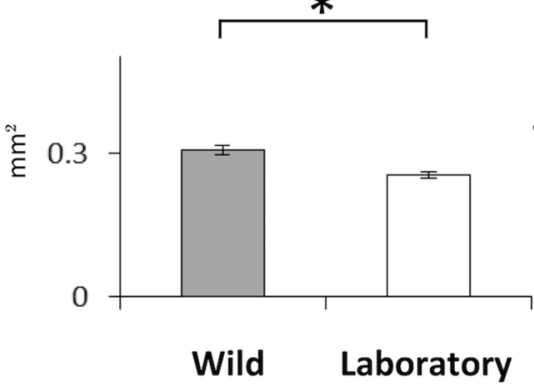

Area of glomerulus layer $*$

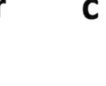

c

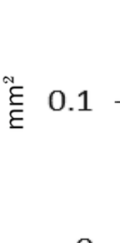

0

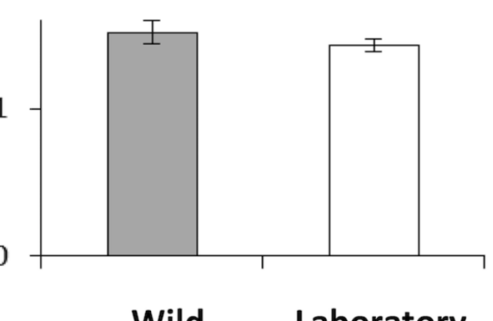

Area of rostral part

Wild

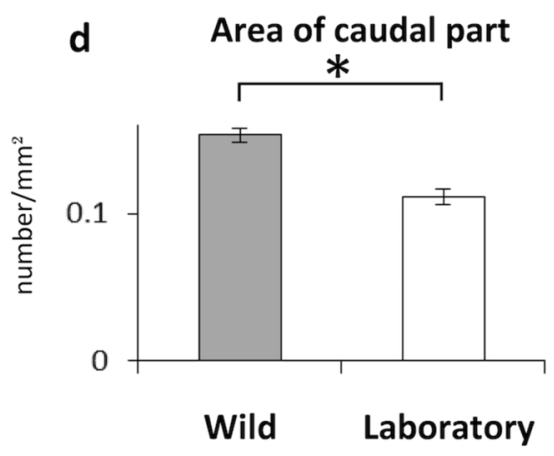

Fig. 3. Anatomical analyses of the accessory olfactory bulb (AOB). (A) A representative AOB section photograph of wild and laboratory rats. The horizontal bar shows $2 \mathrm{~mm}$. The area of (B) whole glomerulus layer and (C) rostral and (D) caudal part of the glomerulus layer in wild (Wild) and laboratory rats (Laboratory). ${ }^{*} P<0.05$ with Student's $t$-test.

(Fig. 3B). We then divided the GL into the Gia-2 immunoreactive rostral part and the remaining caudal part [34], and measured the area of each. We found that the caudal part was significantly larger in the wild rats compared with laboratory rats $(P<0.01)$ whereas we found no significant difference in the rostral part (Fig. 3C and 3D). This finding suggests that the difference in the whole GL was caused by a difference in the caudal part.

\section{DISCUSSION}

The results of the current study revealed that wild rats had significantly larger BLA, BNST, and caudal part of the AOB, compared with laboratory rats. However, we did not find any significant differences in the rostral part of the AOB or in the MOB. Based on these structural differences, we propose that the BLA, BNST, and the vomeronasal system using V2R contribute to wariness in wild rats.

Based on the present findings, it is possible that the BLA in wild rats is more sensitive to stimuli, compared with laboratory rats. This possibility is supported by a recent study using knockout mice lacking a protein that is enriched in the postsynaptic density of excitatory synapses (RICH2) [29]. In that study, the baseline activity of BLA was found to be no different between intact and knockout mice, as assessed by the expression of immediate-early genes (c-Fos and ARC proteins) and their transcription factor (the ratio of phosphorylated CREB/CREB). However, knockout mice, but not intact mice, exhibited avoidance of novel objects with increased Fos expression in the BLA. These findings suggest that differences in sensitivity in the BLA are associated with differences in wariness. The potential role of the BLA appears not to be limited to wariness. Wild rats are known to show different behavioral responses compared with laboratory rats in a range of situations. For example, wild rats, but not laboratory rats, have 
been reported to escape and/or bite experimenters' hands, preventing experimenters from picking them up [37]. In addition, one early study found that when a mouse was placed in the home cage, $70 \%$ of wild rats killed the mouse, whereas only $12 \%$ of laboratory rats exhibited this behavior [14]. Given that BLA-lesion surgery in wild rats is reported to extinguish these differences $[9,14,37]$, the BLA appears to contribute to these behavioral differences. Taken together, it is possible that the BLA in wild rats exhibit greater activation in response to the same stimuli compared with the BLA in laboratory rats.

In the BLA, the density of neurons was higher in wild rats than in laboratory rats. Based on previous findings, we believe that a difference in genetic factors, rather than environmental factors, was likely to be the cause of this difference. It has been reported that the descendants of wild rats maintain the behavioral characteristics of progenitors, even when they are born and reared in the laboratory $[6,9]$. In addition, several genetic factors were reported to affect the size of the BLA and/or the density of neurons in the BLA. For example, a study using knockout mice suggested that a transcription factor involved in neurogenesis (neuroD2) dosedependently increased the number of neurons in the BLA without affecting the neuronal populations in the other nuclei or apoptosis in the BLA [20]. Consistent with this finding, comparison across 35 BXD recombinant inbred mouse strains revealed that the whole-brain expression level of NeuroD2 correlated with the density of neurons in the BLA and volume of the BLA [23]. Similarly, the number of excitatory neurons in the BLA was specifically reduced by apoptosis when the mice lacked a zinc finger gene (Fezf 2$)$ that plays an important role in forming the normal shape of the BLA during prenatal development [11]. Therefore, it is possible that differences in these genetic factors contribute to the difference in the density of neurons between wild and laboratory rats.

The present findings simultaneously suggest that the BNST contributes to wariness in wild rats. This notion is further supported by a previous study reporting that a strain of roof rats that exhibited stronger avoidance of novel objects had an enlarged BNST compared with another strain of roof rats that showed weaker avoidance of novel objects [17]. However, in contrast to the BLA, little attention has been paid to the BNST of wild animals. Future analyses focusing on the role of the BNST are necessary to clarify this point.

In the MOB, we found no differences between wild and laboratory rats, inconsistent with our hypothesis. In contrast, in accord with our hypothesis, we found that the caudal part of the AOB was larger in wild rats compared with laboratory rats. These results suggest that the functioning of the vomeronasal system using V2R is higher in wild rats than in laboratory rats. However, to date, no functional differentiation has been reported between the vomeronasal system using V1R and that using V2R, with one exception: the systems using V1R and V2R were found to detect volatile and non-volatile substances, respectively. Therefore, one possibility is that the increased function of the vomeronasal system using V2R enables wild rats to detect a wider range and/ or weaker intensity of non-volatile olfactory threat stimuli, compared with laboratory rats. Although knowledge of laboratory rats is limited, previous studies have demonstrated that laboratory mice detected predatory threats using V2R, and exhibited avoidance behaviors in response to cat fur or cat saliva [13, 24, 27]. Alternatively, based on the major role of V2R in laboratory mice, it is possible that wild rats can communicate with conspecifics more effectively than laboratory rats. It is known that V2R can detect numerous peptide and protein pheromones that affect aggressive behavior, attraction of females, sexual behavior, and reproduction [26]. In this case, the increased functioning of the vomeronasal system using V2R did not contribute to wariness in wild rats. Further research is required to clarify this point in detail.

In the present study, we found that the sizes of the BLA, BNST, and the caudal part of the AOB were significantly different between the wild and laboratory rats. Based on these findings, we propose that the BLA, BNST, and the caudal part of the AOB contribute to wariness in wild rats. This could be further confirmed by future studies assessing the relationship between the difference in neural activities in these nuclei and differences in each defensive behavior. Domestic animals are thought to lose some behavioral characteristics during the domestication process, even if these behaviors appear to be important for life in the wild [28]. However, because research has mainly focused on a few species of domestic animal, such as laboratory rats and mice, our knowledge of these important characteristics is relatively limited. In addition, we cannot exclude the possibility that the findings observed in domestic animals are not ubiquitous across all wild animals. Future studies conducting behavioral neuroscientific analyses of wild animals will enable us to further our understanding of the relationship between brain and behavior in mammals.

ACKNOWLEDGMENTS. This work was supported by the Japan Society for the Promotion of Science KAKENHI (Grant Numbers $15 \mathrm{H} 05635$ and 15H05782).

\section{REFERENCES}

1. Boreman, J. and Price, E. 1972. Social dominance in wild and domestic Norway rats (Rattus norvegicus). Anim. Behav. 20: 534-542. [CrossRef]

2. Breitfeld, T., Bruning, J. E., Inagaki, H., Takeuchi, Y., Kiyokawa, Y. and Fendt, M. 2015. Temporary inactivation of the anterior part of the bed nucleus of the stria terminalis blocks alarm pheromone-induced defensive behavior in rats. Front. Neurosci. 9: 321. [Medline] [CrossRef]

3. Brubaker, A. S. and Coss, R. G. 2015. Evolutionary constraints on equid domestication: Comparison of flight initiation distances of wild horses (Equus caballus ferus) and plains zebras (Equus quagga). J. Comp. Psychol. 129: 366-376. [Medline] [CrossRef]

4. Chitty, D. and Shorten, M. 1946. Techniques for the study of the Norway rat. J. Mammal. 27: 63-78. [Medline] [CrossRef]

5. Choi, J. S. and Kim, J. J. 2010. Amygdala regulates risk of predation in rats foraging in a dynamic fear environment. Proc. Natl. Acad. Sci. U.S.A. 107: 21773-21777. [Medline] [CrossRef]

6. Cowan, P. E. 1977. Neophobia and neophilia: new-object and new-place reactions of three Rattus species. J. Comp. Physiol. Psychol. $91: 63-71$. [CrossRef]

7. Fendt, M., Endres, T. and Apfelbach, R. 2003. Temporary inactivation of the bed nucleus of the stria terminalis but not of the amygdala blocks freezing induced by trimethylthiazoline, a component of fox feces. J. Neurosci. 23: 23-28. [Medline] [CrossRef] 
8. Fendt, M., Endres, T., Lowry, C. A., Apfelbach, R. and McGregor, I. S. 2005. TMT-induced autonomic and behavioral changes and the neural basis of its processing. Neurosci. Biobehav. Rev. 29: 1145-1156. [Medline] [CrossRef]

9. Galef, B. G. Jr. 1970. Aggression and timidity: responses to novelty in feral Norway rats. J. Comp. Physiol. Psychol. 70: 370-381. [Medline] [CrossRef]

10. Gungor, N. Z. and Paré, D. 2016. Functional Heterogeneity in the Bed Nucleus of the Stria Terminalis. J. Neurosci. 36: 8038-8049. [Medline] [CrossRef]

11. Hirata-Fukae, C. and Hirata, T. 2014. The zinc finger gene Fezf2 is required for the development of excitatory neurons in the basolateral complex of the amygdala. Dev. Dyn. 243: 1030-1036. [Medline] [CrossRef]

12. Inagaki, H., Kiyokawa, Y., Tamogami, S., Watanabe, H., Takeuchi, Y. and Mori, Y. 2014. Identification of a pheromone that increases anxiety in rats. Proc. Natl. Acad. Sci. U.S.A. 111: 18751-18756. [Medline] [CrossRef]

13. Isogai, Y., Si, S., Pont-Lezica, L., Tan, T., Kapoor, V., Murthy, V. N. and Dulac, C. 2011. Molecular organization of vomeronasal chemoreception. Nature 478: 241-245. [Medline] [CrossRef]

14. Karli, P. 1956. The Norway rat's killing response to the white mouse: an experimental analysis. Behaviour 10: 81-103. [CrossRef]

15. Kiyokawa, Y. 2017. Social Odors: Alarm Pheromones and Social Buffering. Curr. Top. Behav. Neurosci. 30: 47-65. [Medline] [CrossRef]

16. Kiyokawa, Y., Mikami, K., Mikamura, Y., Ishii, A., Takeuchi, Y. and Mori, Y. 2015. The 3-second auditory conditioned stimulus is a more effective stressor than the 20-second auditory conditioned stimulus in male rats. Neuroscience 299: 79-87. [Medline] [CrossRef]

17. Kiyokawa, Y., Tanaka, K. D., Ishii, A., Mikami, K., Katayama, M., Koizumi, R., Minami, S., Tanikawa, T. and Takeuchi, Y. 2017. Two strains of roof rats as effective models for assessing new-object reaction. J. Vet. Med. Sci. 79: 702-708. [Medline] [CrossRef]

18. LeDoux, J. 2012. Rethinking the emotional brain. Neuron 73: 653-676. [Medline] [CrossRef]

19. Liberles, S. D. 2014. Mammalian pheromones. Annu. Rev. Physiol. 76: 151-175. [Medline] [CrossRef]

20. Lin, C. H., Hansen, S., Wang, Z., Storm, D. R., Tapscott, S. J. and Olson, J. M. 2005. The dosage of the neuroD2 transcription factor regulates amygdala development and emotional learning. Proc. Natl. Acad. Sci. U.S.A. 102: 14877-14882. [Medline] [CrossRef]

21. Lõhmus, M., Janse, I., van de Goot, F. and van Rotterdam, B. J. 2013. Rodents as potential couriers for bioterrorism agents. Biosecur. Bioterror. 11 Suppl 1: S247-S257. [Medline] [CrossRef]

22. Mori, K., von Campenhause, H. and Yoshihara, Y. 2000. Zonal organization of the mammalian main and accessory olfactory systems. Philos. Trans. R. Soc. Lond. B Biol. Sci. 355: 1801-1812. [Medline] [CrossRef]

23. Mozhui, K., Hamre, K. M., Holmes, A., Lu, L. and Williams, R. W. 2007. Genetic and structural analysis of the basolateral amygdala complex in BXD recombinant inbred mice. Behav. Genet. 37: 223-243. [Medline] [CrossRef]

24. Papes, F., Logan, D. W. and Stowers, L. 2010. The vomeronasal organ mediates interspecies defensive behaviors through detection of protein pheromone homologs. Cell 141: 692-703. [Medline] [CrossRef]

25. Pêgo, J. M., Morgado, P., Pinto, L. G., Cerqueira, J. J., Almeida, O. F. and Sousa, N. 2008. Dissociation of the morphological correlates of stressinduced anxiety and fear. Eur. J. Neurosci. 27: 1503-1516. [Medline] [CrossRef]

26. Pérez-Gómez, A., Stein, B., Leinders-Zufall, T. and Chamero, P. 2014. Signaling mechanisms and behavioral function of the mouse basal vomeronasal neuroepithelium. Front. Neuroanat. 8: 135. [Medline]

27. Pérez-Gómez, A., Bleymehl, K., Stein, B., Pyrski, M., Birnbaumer, L., Munger, S. D., Leinders-Zufall, T., Zufall, F. and Chamero, P. 2015. Innate Predator Odor Aversion Driven by Parallel Olfactory Subsystems that Converge in the Ventromedial Hypothalamus. Curr. Biol. 25: 1340-1346. [Medline] [CrossRef]

28. Rauw, W. M., Johnson, A. K., Gomez-Raya, L. and Dekkers, J. C. M. 2017. A Hypothesis and Review of the Relationship between Selection for Improved Production Efficiency, Coping Behavior, and Domestication. Front. Genet. 8: 134. [Medline] [CrossRef]

29. Sarowar, T., Grabrucker, S., Boeckers, T. M. and Grabrucker, A. M. 2017. Object Phobia and Altered RhoA Signaling in Amygdala of Mice Lacking RICH2. Front. Mol. Neurosci. 10: 180. [Medline] [CrossRef]

30. Shackman, A. J. and Fox, A. S. 2016. Contributions of the Central Extended Amygdala to Fear and Anxiety. J. Neurosci. 36: 8050-8063. [Medline] [CrossRef]

31. Staples, L. G. 2010. Predator odor avoidance as a rodent model of anxiety: learning-mediated consequences beyond the initial exposure. Neurobiol. Learn. Mem. 94: 435-445. [Medline] [CrossRef]

32. Takahashi, L. K. 2014. Olfactory systems and neural circuits that modulate predator odor fear. Front. Behav. Neurosci. 8: 72. [Medline] [CrossRef]

33. Takahashi, L. K., Hubbard, D. T., Lee, I., Dar, Y. and Sipes, S. M. 2007. Predator odor-induced conditioned fear involves the basolateral and medial amygdala. Behav. Neurosci. 121: 100-110. [Medline] [CrossRef]

34. Takigami, S., Mori, Y., Tanioka, Y. and Ichikawa, M. 2004. Morphological evidence for two types of Mammalian vomeronasal system. Chem. Senses 29: 301-310. [Medline] [CrossRef]

35. Walker, D. L. and Davis, M. 1997. Double dissociation between the involvement of the bed nucleus of the stria terminalis and the central nucleus of the amygdala in startle increases produced by conditioned versus unconditioned fear. J. Neurosci. 17: 9375-9383. [Medline] [CrossRef]

36. Whishaw, I. Q. 1999. The laboratory rat, the Pied Piper of twentieth century neuroscience. Brain Res. Bull. 50: 411. [Medline] [CrossRef]

37. Woods, J. W. 1956. Taming of the wild Norway rat by rhinencephalic lesions. Nature 178: 869. [Medline] [CrossRef] 